\title{
Analysis of Digital Modulation with Unequal Power Allocation
}

\author{
Thomas Brüggen and Peter Vary \\ Institute of Communication Systems and Data Processing (indl), RWTH Aachen University, Germany \\ \{brueggen,vary\}@ind.rwth-aachen.de
}

\begin{abstract}
In digital transmission systems for speech, audio, and video signals source encoders extract parameters which are quantized and converted into a digital representation. As the individual bits of this representation exhibit different bit error sensitivities, usually channel coding with unequal error protection (UEP) is applied. However, some transmission systems do not include channel coding for several reasons. In this situation a concept called modulation with unequal power allocation (MUPA) can be applied which achieves UEP by allocating different transmission power to the modulation symbols according to the individual bit error sensitivities. The average transmitted energy per bit remains unaffected. In this contribution we present a new detailed analysis of BPSK-MUPA and 16-QAM-MUPA, and discuss the performance improvements in terms of parameter SNR compared to systems with constant symbol energy.
\end{abstract}

\section{INTRODUCTION}

Unequal error protection (UEP) is often realized by selective channel coding [1]. However, some transmission systems for speech, audio, or video signals allow only weak or no channel coding (e.g., DECT, Bluetooth). For this kind of systems, we analyze the novel concept with implicit UEP by modulation with unequal power allocation (MUPA) [2],[3]. It distributes the transmission energy unequally in a periodically time-varying manner to the modulation signal points by the so-called weights $w_{i}, i=1,2, \ldots M$ with $M$ being the number of bits in a frame, e.g., the bits assigned to a quantizer reproduction level. The weights influence the distances between the signal points. The average transmitted energy per bit remains unaffected. According to the mapping of the bit patterns, the distance between modulation signal points which differ in the most significant bit (MSB) is made larger. Other distances between signal points differing, e.g., in the least significant bit (LSB) are made smaller. For each channel $E_{b} / N_{0}$ a set of weights is calculated once in advance.

UEP by differently weighted bits has already been discussed in [4] and [5]. Differently weighted bits in pulse code modulation (PCM) transmission have been discussed in [4]. However, an approximate solution only for PCM is derived which is based on the simplifying assumption that only single bit errors occur. In [5] discrete symbol amplitudes are calculated for BPSK with a fixed step size. In contrast to [4] and [5], the MUPA approach allows rigorous optimization of the continuous symbol amplitudes and is derived in [3] for BPSK and in [2] for 16-QAM. [3] and [2] are extended in this contribution by a new detailed examination of the optimized weights for BPSK-MUPA and 16-QAM-MUPA, and the performance improvements compared to systems with constant symbol energy. As optimization criterion we consider the mean square error (MSE) $\mathrm{E}\left\{(\mathrm{u}(\tau)-\hat{\mathrm{u}}(\tau))^{2}\right\}$ in the parameter domain. The corresponding signal-to-noise ratio (SNR) between the original codec parameters $u(\tau)$ (Figs. 1 and 3) and its reconstruction $\hat{u}(\tau)$ is given by

$$
\mathrm{SNR}[\mathrm{dB}]=10 \log _{10}\left(\frac{\mathrm{E}\left\{\mathrm{u}^{2}(\tau)\right\}}{\mathrm{E}\left\{(\mathrm{u}(\tau)-\hat{\mathrm{u}}(\tau))^{2}\right\}}\right) .
$$

As the parameter SNR has a strong correlation with the audio or video quality, we utilize it to compare the performance of MUPA with modulation schemes using fixed signal constellations. Additionally, we analyze the influence of a weight mismatch with respect to the $E_{b} / N_{0}$ estimation.

The paper is organized as follows. Firstly, the baseband system models for BPSK and 16-QAM are described. In Sec. IV the optimization approach and its solution with the Lagrange Multiplier method and the Newton algorithm is explained for BPSK first, and then for 16-QAM in a second step. The analysis of the optimized weights follows. Finally, simulation results demonstrate the parameter SNR gains for BPSK-MUPA and 16-QAM-MUPA compared to systems with constant symbol energy.

\section{BPSK SYSTEM MODEL}

The model of the BPSK transmission system is shown in Fig. 1. Instead of any specific signal and source encoder, we use a zero-mean Gaussian source with variance $\sigma_{u}^{2}=1$ to model the parameters $u(\tau)$ which are delivered at time $\tau$ by the source encoder. This allows us to conduct precisely defined experiments. However, without loss of generality the results can be applied to any real source codec. Each $u(\tau)$ is quantized to $\bar{u}(\tau) \in\left\{\bar{u}_{\kappa} \mid \kappa=1,2, \ldots 2^{M}\right\}$ with the reproduction levels $\bar{u}_{\kappa}$. The index assignment (IA) utilitzes the one-to-one mapping function $\Gamma$ and maps the values $\bar{u}(\tau)$ to the bit patterns $\underline{x}(\tau) \in\left\{\underline{x}_{\kappa} \mid \kappa=1,2, \ldots 2^{M}\right\}$ with $\underline{x}_{\kappa}=\left(x_{\kappa}^{(1)}, x_{\kappa}^{(2)}, \ldots x_{\kappa}^{(M)}\right)$ :

$$
\underline{x}(\tau)=\Gamma(\bar{u}(\tau)) .
$$

The bits $x_{\kappa}^{(i)} \in\{-1,+1\}, i=1,2, \ldots M$, are ordered from the MSB $x_{\kappa}^{(1)}$ to the LSB $x_{\kappa}^{(M)}$. Each value $x_{\kappa}^{(i)}$ is multiplied with the specific weight $w_{i} \in \mathbb{R}^{+}$(Sec. IV) of the diagonal matrix

$$
\underline{W}=\operatorname{diag}\left(w_{1}, w_{2}, \ldots w_{M}\right) .
$$

The obtained channel symbols (Fig. 2)

$$
\underline{y}(\tau)=\underline{W} \cdot \underline{x}(\tau)
$$




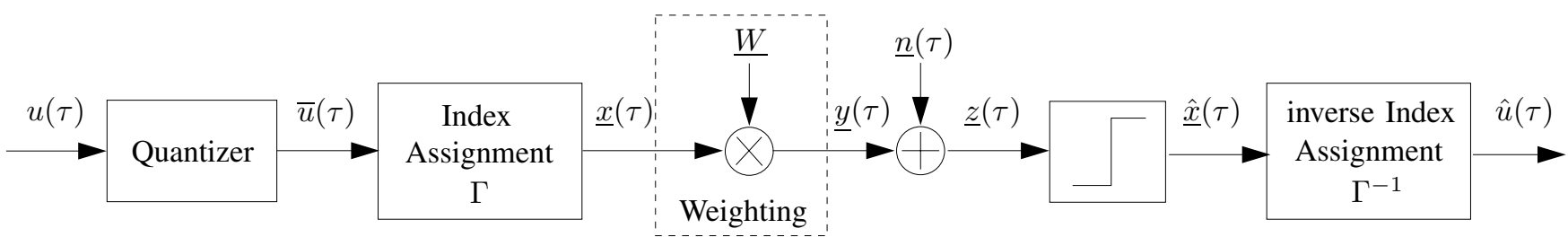

Fig. 1. Baseband system model of BPSK-MUPA with weight matrix $\underline{W}$ and hard decision decoding

are transmitted over a channel with additive white Gaussian noise (AWGN) $\underline{n}(\tau)=\left(n^{(1)}(\tau), n^{(2)}(\tau), \ldots n^{(M)}(\tau)\right)$, $n^{(i)}(\tau) \in \mathbb{R}, i=1,2, \ldots M$, with the distribution $N\left(0, \sigma_{n}^{2}\right)$, the variance $\sigma_{n}^{2}=N_{0} / 2$, and the noise power spectral density $N_{0}$.

In case of coherent BPSK modulation we obtain the disturbed vector $\underline{z}(\tau)=\underline{y}(\tau)+\underline{n}(\tau)$ at the receiver. After hard decision decoding, the estimate $\hat{u}(\tau) \in\left\{\bar{u}_{\kappa} \mid \kappa=1,2, \ldots 2^{M}\right\}$ is produced by inverse IA (table lookup).

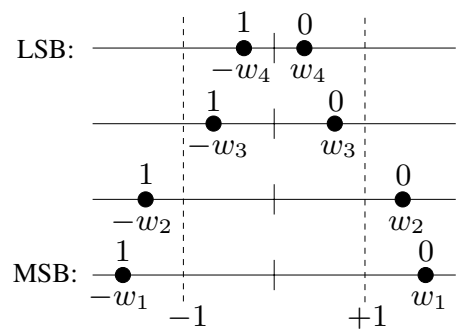

Fig. 2. Channel symbols $y(\tau)$ for BPSK-MUPA with their amplitudes $w_{i}$ for $M=4$ bits, binary bit representation

\section{16-QAM SYSTEM MODEL}

The baseband model of the 16-QAM transmission system is shown in Fig. 3. Source, quantizer and index assignment are the same as for BPSK-MUPA (Sec. II). In contrast to BPSK, the modulation signal points of the 16-QAM scheme consist of an inphase (I) component and a quadrature (Q) component. While 1 bit per modulation symbol is transmitted in the BPSK scheme, the modulation signal points of 16-QAM are assigned to 4 bits. The 16-QAM signal points are shifted by the weights according to the differing bits (Fig. 4).

After the IA, the modulator assigns each bit pattern $\underline{x}(\tau)$ by the one-to-one mapping function $\mu$ to a complex modulation signal point $y(\tau)$ of the signal constellation set $\mathcal{Y}=\left\{y_{\kappa} \mid \kappa=1,2, \ldots 2^{M}\right\}$ :

$$
y(\tau)=\mu(\underline{x}(\tau))=y^{(I)}(\tau)+\mathrm{j} y^{(Q)}(\tau)
$$

with the inphase (I) component $y^{(I)}(\tau)$ and the quadrature (Q) component $y^{(Q)}(\tau)$ of $y(\tau)$. The transmission of the obtained complex symbols $y(\tau) \in \mathcal{Y}$ is described by a complex channel with zero-mean additive white Gaussian noise (AWGN) $n(\tau)=n^{(I)}(\tau)+\mathrm{j} n^{(Q)}(\tau)$ with $n^{(I)}(\tau) \in \mathbb{R}$ in the inphase dimension and $n^{(Q)}(\tau) \in \mathbb{R}$ in the quadrature dimension. We consider an AWGN channel with independent transmission of the two components with the variance $\sigma_{n}^{2}=N_{0} / 2$ in each dimension and the noise power spectral density $N_{0}$. The average signal point energy $\mathrm{E}\left\{E_{y}\right\}$ of all $y_{\kappa} \in \mathcal{Y}$, $\kappa=1,2, \ldots 2^{M}$, is normalized to $\mathrm{E}\left\{E_{y}\right\}=M$ to ensure an average energy per bit $E_{b}$ of 1 .

At the receiver, the symbol is estimated from the disturbed complex samples $z(\tau)=\left(y^{(I)}(\tau)+n^{(I)}(\tau)\right)+\mathrm{j}\left(y^{(Q)}(\tau)+\right.$ $\left.n^{(Q)}(\tau)\right)$ by hard decision demodulation. The inverse IA returns the estimated $\hat{u}(\tau)$ corresponding to the estimated quantized value $\bar{u}(\tau)$ by a codebook table lookup.

\section{Unequal Power Allocation}

Since the MUPA algorithm [2],[3] is the subject of our analysis in Sec. VI, we review it in the following. From now on, we assume ergodicity and perfect knowledge of $\sigma_{n}^{2}$ at the transmitter and at the receiver. The latter condition is alleviated later in Sec. VI.

\section{A. Approach for BPSK}

We consider bipolar channel symbols $\pm \sqrt{E_{b}^{(i)}}$ with $E_{b}^{(i)}$ being the energy for each bit $i$. If bits in $\underline{x}(\tau)$ are inverted due to AWGN, a wrong decision $\underline{\hat{x}}(\tau)$ is made at the receiver. Thus, the distortion $d(\tau)=\bar{u}(\tau)-\hat{u}(\tau)$ occurs. We want to calculate optimal weights in the sense of a minimized expected value $\mathrm{E}\left\{d^{2}(\tau)\right\}$.

In the following we skip the time index $\tau$ due to ergodicity. Thus, we calculate $\mathrm{E}\left\{d^{2}\right\}$ taking the different possible values

$$
d_{\kappa, \eta}=\bar{u}_{\kappa}-\hat{u}_{\eta}
$$

with $\kappa, \eta=1,2, \ldots 2^{M}$ into account:

$$
\mathrm{E}\left\{d^{2}\right\}=\sum_{\kappa=1}^{2^{M}} \sum_{\eta=1}^{2^{M}} d_{\kappa, \eta}^{2} \cdot P\left(\underline{x}_{\kappa}\right) \cdot P\left(\underline{\hat{x}}_{\eta} \mid \underline{x}_{\kappa}\right)
$$

The MSE E $\left\{d^{2}\right\}$ depends on the probability of occurrence $P\left(\underline{x}_{\kappa}\right)$ of the reproduction levels $\bar{u}_{\kappa}$ and on the transition probabilities $P\left(\underline{\hat{x}}_{\eta} \mid \underline{x}_{\kappa}\right)$ between the transmitted and received bit patterns $\underline{x}_{\kappa}$ to $\underline{\hat{x}}_{\eta}$, respectively.

MUPA with all $w_{i}=1.0, i=1, \ldots M$, is identical to BPSK with equal bit error rates (BER) $P_{b}$ for all bits [6]

$$
P_{b}=\frac{1}{2} \operatorname{erfc}\left(\sqrt{\frac{E_{b}}{N_{0}}}\right)=\int_{\sqrt{E_{b}}}^{\infty} \frac{1}{\sqrt{2 \pi} \sigma_{n}} \cdot \exp \left(\frac{-\xi^{2}}{2 \sigma_{n}^{2}}\right) \mathrm{d} \xi .
$$

$P_{b}$ depends on the bit energy $E_{b}$. With MUPA, the $M$ different bits are transmitted with different bit energies $E_{b}^{(i)}=w_{i}^{2} \cdot E_{b}$, 


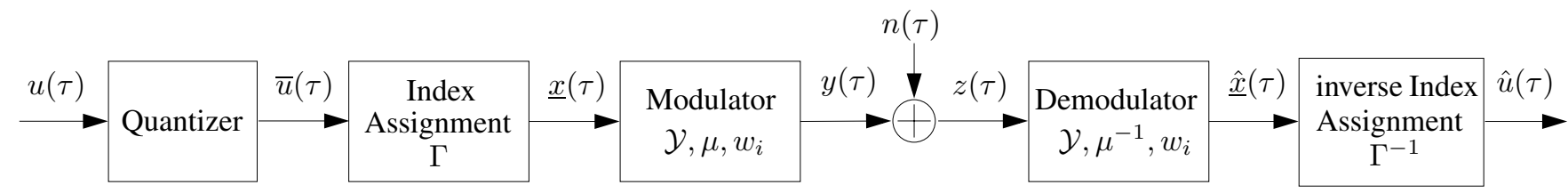

Fig. 3. Baseband system model of 16-QAM-MUPA with hard demodulation

$i=1, \ldots M$. If $w_{i}>1$, the bit error probability is reduced due to the higher amplitude, i.e., higher energy for bit $i$. Otherwise, if $0<w_{i}<1$, the BER is increased. This different weighting is illustrated in Fig. 2.

In most transmission systems the energy budget is limited or additional signal energy increases interference as, e.g., in code division multiple access (CDMA) systems. We restrict the transmission energy by normalizing the average bit energy $E_{b}$ to 1 . With the different bit energies $E_{b}^{(i)}=w_{i}^{2}$ the energy constraint is given by

$$
\sum_{i=1}^{M} w_{i}^{2}=M
$$

and the different bit error probabilities by

$$
P_{b}^{(i)}=\int_{w_{i}}^{\infty} \frac{1}{\sqrt{2 \pi} \sigma_{n}} \cdot \exp \left(\frac{-\xi^{2}}{2 \sigma_{n}^{2}}\right) \mathrm{d} \xi .
$$

As the noise samples are statistically independent, the transition probabilities can be calculated as [3],[6],[7]

$$
P\left(\underline{\hat{x}}_{\eta} \mid \underline{x}_{\kappa}\right)=\left(\prod_{\substack{i=1 \\ x_{\kappa}^{(i)} \neq \hat{x}_{\eta}^{(i)}}}^{M} P_{b}^{(i)}\right) \cdot\left(\prod_{\substack{i=1 \\ x_{\kappa}^{(i)}=\hat{x}_{\eta}^{(i)}}}^{M}\left(1-P_{b}^{(i)}\right)\right) .
$$

\section{B. Lagrange Multiplier Method (LMM)}

Our goal is to minimize the MSE E $\left\{d^{2}\right\}$ of (7) by optimizing the weights $w_{i}$ under the constraint (9). An appropriate approach to find a solution for this optimization problem is the Langrange Multiplier Method (LMM) with the Lagrange Multiplier $\lambda$. Applying (7), (9) and (11) yields to the LMM approach:

$$
\begin{gathered}
L\left(w_{1}, \ldots w_{M}, \lambda\right)= \\
\left(\sum_{\kappa=1}^{2^{M}} \sum_{\eta=1}^{2^{M}} d_{\kappa, \eta}^{2} \cdot P\left(\underline{x}_{\kappa}\right) \cdot\left(\prod_{\substack{i=1 \\
x_{\kappa}^{(i)} \neq \hat{x}_{\eta}^{(i)}}}^{M} P_{b}^{(i)}\right) \cdot\left(\prod_{\substack{i=1 \\
x_{\kappa}^{(i)}=\hat{x}_{\eta}^{(i)}}}^{M}\left(1-P_{b}^{(i)}\right)\right)\right) \\
-\lambda\left(M-\sum_{i=1}^{M} w_{i}^{2}\right) .
\end{gathered}
$$

This equation is a non-linear function $L: \mathbb{R}^{M+1} \rightarrow \mathbb{R}$ with $M+1$ unknown variables, i.e., the $M$ weights $w_{i}$ and the Lagrange Multiplier $\lambda$. Next, we need the $M+1$ partial derivatives $\partial L\left(w_{1}, \ldots w_{M}, \lambda\right) / \partial w_{j}, j=1,2, \ldots M$, and $\partial L\left(w_{1}, \ldots w_{M}, \lambda\right) / \partial \lambda$ of (12).

\section{Newton Algorithm}

In the next LMM step the roots $w_{j}$ and $\lambda$ of the $M+1$ derivatives have to be calculated. Due to the transcendent function $\exp (x)$, we employ the $(M+1)$-dimensional Newton algorithm to calculate the roots. For convenience, we insert $\lambda$ into the set of variables by renaming $\left(w_{1}, \ldots w_{M}, \lambda\right)^{T}$ to $\underline{v}=\left(v_{1}, \ldots v_{M+1}\right)^{T}$ with $v_{i}=w_{i}$ for $i=1,2, \ldots M$, and $v_{M+1}=\lambda$. We define $L_{j}^{\prime}(\underline{v})=\partial L(\underline{v}) / \partial v_{j}$, $j=1,2, \ldots M+1$, and $\underline{L}^{\prime}(\underline{v})=\left(L_{1}^{\prime}(\underline{v}), \ldots L_{M+1}^{\prime}(\underline{v})\right)^{T}$ for the vector of the first partial derivatives. With this notation, the Newton iteration rule is given by

$$
\underline{v}_{k+1}=\underline{v}_{k}-J_{L}^{-1}\left(\underline{v}_{k}\right) \cdot \underline{L}^{\prime}\left(\underline{v}_{k}\right)
$$

with the iteration counter $k$ and the inverse Jacobian matrix $J_{L}^{-1}\left(\underline{v}_{k}\right)$ containing the first partial derivatives of $L^{\prime}(\underline{v})$, i.e., the second partial derivatives of $L(\underline{v}), L^{\prime \prime}(\underline{v})=\partial L_{g}^{\prime}(\underline{v}) / \partial v_{j}$, for all combinations of $g, j=1,2, \ldots M+1$. The number of iterations can be reduced by adapting $\lambda$ in each iteration $k$ according to the difference between $\underline{v}_{k+1}$ and $\underline{v}_{k}$.

When the exit condition $\left\|\underline{v}_{k+1}-\underline{v}_{k}\right\|<\epsilon$ is fulfilled, the LMM supplies the weights $w_{i}$ as optimized solution. It is very difficult to check this solution for minimum analytically due to the non-linearity of (7) and (9). For simplification, we take other sets of weights with $w_{i}^{*}$ fulfilling (9) and which are close to $w_{i}$. If the $\mathrm{E}\left\{d^{2}\right\}$ of (7) is larger with the $w_{i}^{*}$ than with the $w_{i}$, we suppose that the optimized $w_{i}$ are the solution for a local or a global minimum. The simulation results (Sec. VI) show that this strategy is successful.

\section{Approach for 16-QAM}

As the modulation signal points of 16-QAM are assigned to 4 bits, the MUPA approach has to be transferred to symbol level. In the example of Fig. 4, a conventional 16-QAM scheme with Gray labeling (Fig. 4a) is compared to the modified scheme (Fig. 4b) with unequal weights $w_{i}$ and, e.g.,

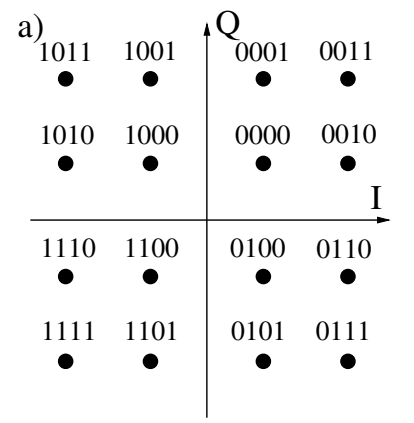

b)

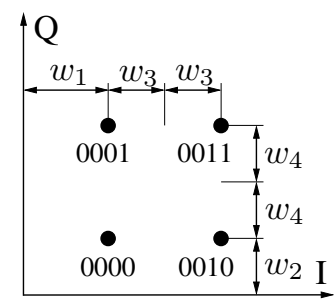

Fig. 4. a) Gray mapped bit patterns of 16-QAM signal points [8] b) first quadrant of a) but with unequal weights $w_{i}$, $w_{1}>w_{2}>w_{3}>w_{4}$, as example for all quadrants 
$w_{1}$ being responsible for the error events of the MSB (e.g., signal points labeled 1001 and 0001).

If the demodulator selects the wrong signal point out of $\mathcal{Y}$ due to AWGN, the distortion $d$ between the quantized source sample and the estimated sample occurs. With the focus on the 16-QAM modulation, $\mathrm{E}\left\{d^{2}\right\}$ depends on the probability of occurrence $P\left(y_{\kappa}\right)$ of the signal points and on the probability that the signal point $y_{\kappa} \in \mathcal{Y}$ was sent and $\hat{y}_{\eta} \in \mathcal{Y}$ with $\hat{y}_{\eta}=\mu\left(\underline{\hat{x}}_{\eta}\right)$ is received, i.e., the transition probability $P\left(\hat{y}_{\eta} \mid y_{\kappa}\right)$. Consequently, the mean square error $\mathrm{E}\left\{d^{2}\right\}$ has to be minimized analog to BPSK-MUPA (7)

$$
\mathrm{E}\left\{d^{2}\right\}=\sum_{\kappa=1}^{2^{M}} \sum_{\eta=1}^{2^{M}} d_{\kappa, \eta}^{2} \cdot P\left(y_{\kappa}\right) \cdot P\left(\hat{y}_{\eta} \mid y_{\kappa}\right) .
$$

The probability of occurrence $P\left(y_{\kappa}\right)$ equals $P\left(\mu\left(\Gamma\left(\bar{u}_{\kappa}\right)\right)\right)$ of the quantizer reproduction levels. The transition probability $P\left(\hat{y}_{\eta} \mid y_{\kappa}\right)$ is the probability, that the channel noise exceeds the real-valued geometric distance from $y_{\kappa}$ to the decision bound between $y_{\kappa}$ and $\hat{y}_{\eta}$, i.e., the weight $w_{i}$ :

$$
P\left(\hat{y}_{\eta} \mid y_{\kappa}\right)=\int_{w_{i}}^{\infty} \frac{1}{\sqrt{2 \pi} \sigma_{n}} \cdot \exp \left(\frac{-\xi^{2}}{2 \sigma_{n}^{2}}\right) \mathrm{d} \xi
$$

with $w_{i}$ corresponding to $\hat{y}_{\eta}$ and $y_{\kappa}$. If the upper limit of the integral is set to infinity, only the nearest neighbor of $y_{\kappa}$ is considered in the case of 16-QAM. Otherwise, the upper limit has to be adjusted according to the specific signal constellation set causing much higher computational effort.

For 16-QAM the average energy $\mathrm{E}\left\{E_{y}\right\}$ per complex symbol $y_{\kappa} \in \mathcal{Y}$ has to be normalized to $M$ :

$$
\mathrm{E}\left\{E_{y}\right\}=\sum_{\kappa=1}^{2^{M}} E_{y}^{(\kappa)} \cdot P\left(y_{\kappa}\right)=M
$$

with the energy $E_{y}^{(\kappa)}=\left\|y_{\kappa}\right\|^{2}, \kappa=1,2, \ldots 2^{M}$, depending on the weights $w_{i}, i=1, \ldots M$.

Analog to BPSK-MUPA, the Lagrange equation combining (14) and (16) on signal point level is given by

$$
\begin{aligned}
L\left(w_{1}, w_{2}, \ldots w_{M}, \lambda\right) & =\left(\sum_{\kappa=1}^{2^{M}} \sum_{\eta=1}^{2^{M}} d_{\kappa, \eta}^{2} \cdot P\left(y_{\kappa}\right) \cdot P\left(\hat{y}_{\eta} \mid y_{\kappa}\right)\right) \\
& -\lambda\left(M-\mathrm{E}\left\{E_{y}\right\}\right) .
\end{aligned}
$$

\section{AnAlysis of Weights}

Fig. 5 shows the optimized weights for BPSK-MUPA. The weights $w_{1}$ and $w_{2}$ for the two MSBs are emphasized at the cost of $w_{3}$ and $w_{4}$. With increasing $E_{b} / N_{0}$ all weights approach towards $w_{i}=1$. The reason is the extreme sensitivity (gradient) of the bit error probability in that range with respect to weight variations. Consequently, the different weights are still different but close to 1 .

The optimized weights for 16-QAM-MUPA (Fig. 6) behave similarly to the ones for BPSK-MUPA. However, for channels with $E_{b} / N_{0} \leq 3 \mathrm{~dB} w_{4}$ is negative. This can be explained

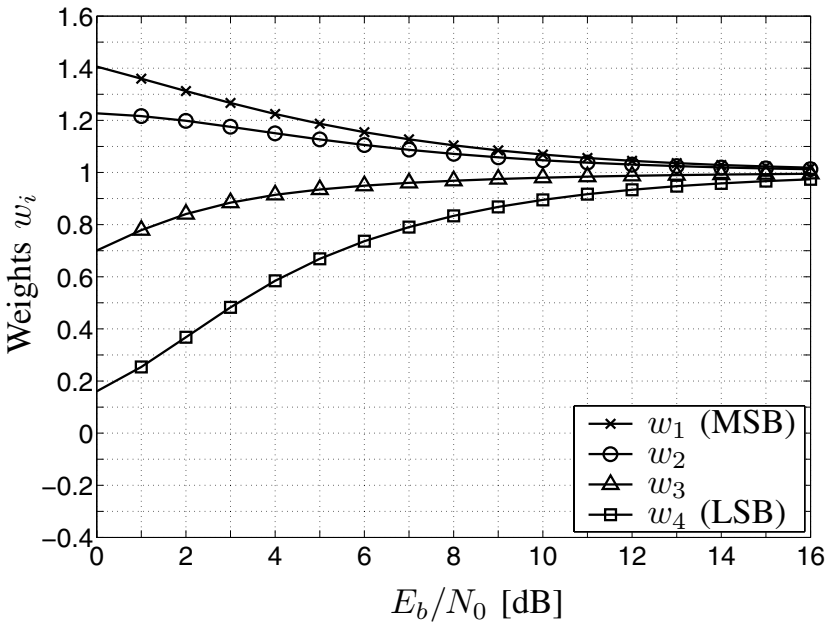

Fig. 5. BPSK-MUPA weight values $w_{i}$ (LMQ, $\Gamma$ : Gray)

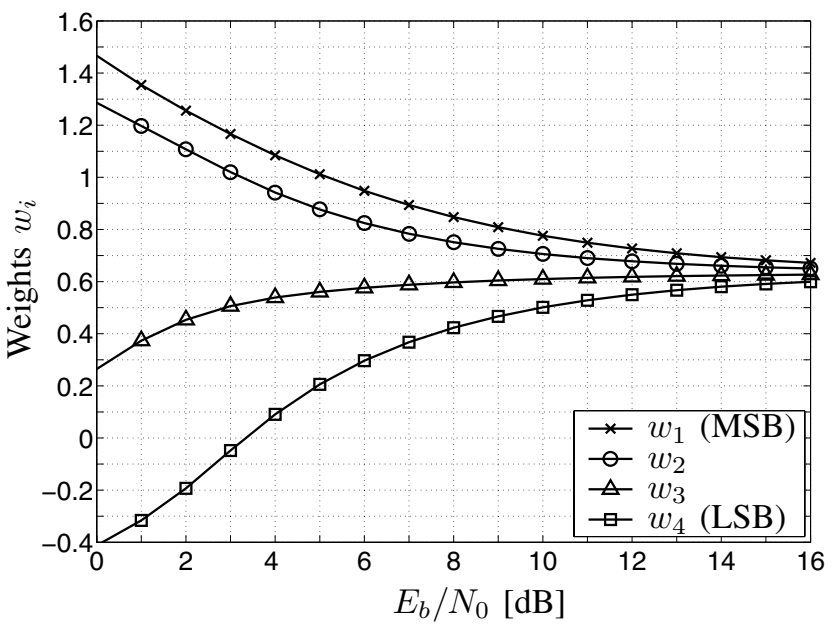

Fig. 6. 16-QAM-MUPA weight values $w_{i}$ (LMQ, $\Gamma: \mathrm{NB}, \mu$ : Gray) with the simplification to consider the next symbol neighbors only, and the energy constraint (16) for 16-QAM-MUPA. The energy $E_{y}^{(\kappa)}$ of the symbol $y_{\kappa}$ depends on the weights $w_{i}$. For example, in Fig. 4 the signal points labeled 1001, 0001, 1101, and 0101 are transmitted with the same energy given by

$$
\left\|y^{2}\right\|=w_{1}^{2}+\left(w_{2}+2 w_{4}\right)^{2} .
$$

In contrast to the energy constraint (9) for BPSK-MUPA, in (18) not all weights occur to the power of 2 . As the Lagrange/Newton algorithm supplies a mathematical optimized solution, the calculated weights have no immediate physical meaning. Negative weights are possible and are not eliminated by the square as in (9). The resulting weights can be adapted to the 16-QAM system of Fig. 4, e.g., by always taking the absolute weight value. In this case the energy constraint (16) is not fulfilled. However, in our transmission system the $2^{M}$ optimized modulation signal points are normalized such that $E_{b}=1$ is ensured on the channel.

Negative weights can only occur at bad channels, because the weights for 16-QAM-MUPA ( $\Gamma: \mathrm{NB}, \mu$ : Gray) approach towards the positive value $w_{i}=2 / \sqrt{10}$ at higher $E_{b} / N_{0}$ values due to (16).

The influence of BPSK-MUPA on the BER is depicted in Fig. 7. According to the significance of the bits, the bit 


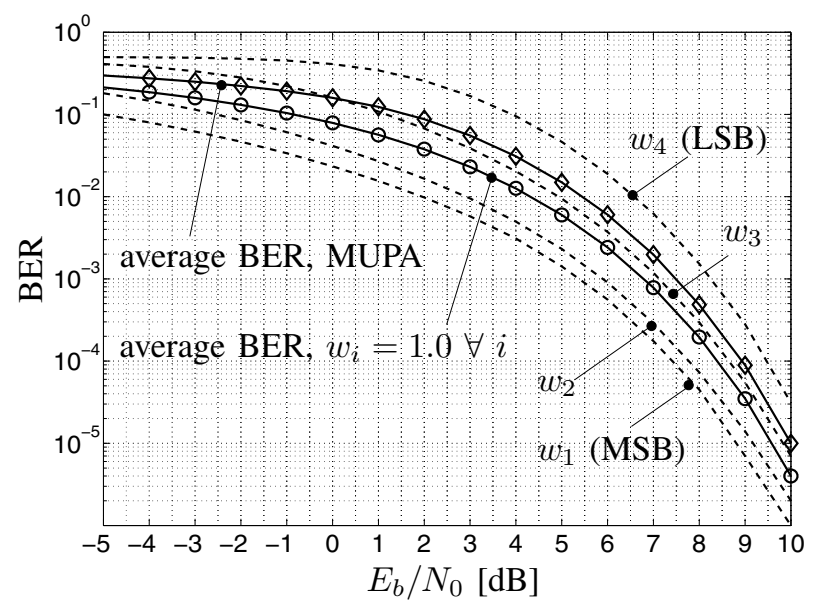

Fig. 7. Bit error rates of BPSK-MUPA ( $\Gamma$ : Gray)

specific BERs are distributed above (LSB) and below (MSB) the average BER curve. Due to the high BER of the LSB $w_{4}$, the average BER is worse with MUPA than without, i.e., $w_{i}=1.0 \forall i$. However, the parameter SNR, i.e., the hearing impression of an audio file, becomes better with MUPA (Sec. VI).

\section{Simulation Results}

The performance of MUPA is evaluated in terms of the parameter SNR (1) and confirmed by simulations with

- a source signal with zero-mean Gaussian probability density function (pdf), the variance $\sigma_{u}^{2}=1$,

- a symmetric Lloyd-Max quantizer (LMQ) which is pdfoptimized for the source signal,

- BPSK with index assignment $\Gamma$ : Gray,

- 16-QAM modulation scheme of Fig. 4 with $\Gamma$ : natural binary (NB) and $\mu$ : Gray.

Figs. 8 and 9 show the performance improvements of BPSK-MUPA and 16-QAM-MUPA (solid lines marked "o"), respectively, compared to the corresponding systems with constant symbol energy (dashed lines). The maximum parameter SNR gain (vertical gain) for BPSK-MUPA is $2.61 \mathrm{~dB}$ at $E_{b} / N_{0}=2 \mathrm{~dB}$, and for 16-QAM-MUPA $3.55 \mathrm{~dB}$ at $E_{b} / N_{0}=5 \mathrm{~dB}$.

So far, perfect knowledge of $\sigma_{n}^{2}$ at the transmitter and the receiver has been assumed. However, MUPA is quite insensitive to $E_{b} / N_{0}$ mismatches within specific limits. The curves for the weight mismatch are also depicted in Figs. 8 and 9, i.e., the transmitter and the receiver use the same set of weights of the $E_{b} / N_{0}$ values $-3,0,3,5$, and $10 \mathrm{~dB}$, respectively, for all channel qualities. As a result, the weights of a higher $E_{b} / N_{0}$ should be used to reduce the performance loss, if the $E_{b} / N_{0}$ cannot be estimated exactly. For example, if the channel quality is only approximately known and somewhere in the $E_{b} / N_{0}$ range of 3 to $6 \mathrm{~dB}$, the weights optimized for $E_{b} / N_{0}=5 \mathrm{~dB}$ should be used instead of the set of weights for $E_{b} / N_{0}=3 \mathrm{~dB}$.

\section{CONCLUSION}

Modulation with unequal power allocation (MUPA) adjusts the distances between the modulation signal points accord-

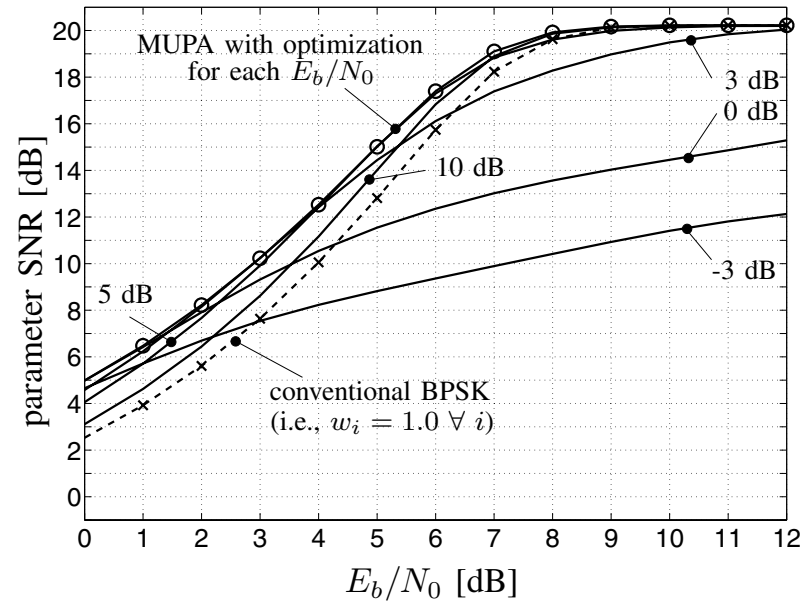

Fig. 8. Comparison of BPSK ( $\Gamma$ : Gray) with and without MUPA, and cases of weight mismatch (weights optimized for the $E_{b} / N_{0}$ values -3 , $0,3,5$, and $10 \mathrm{~dB}$ are used for all channel qualities).

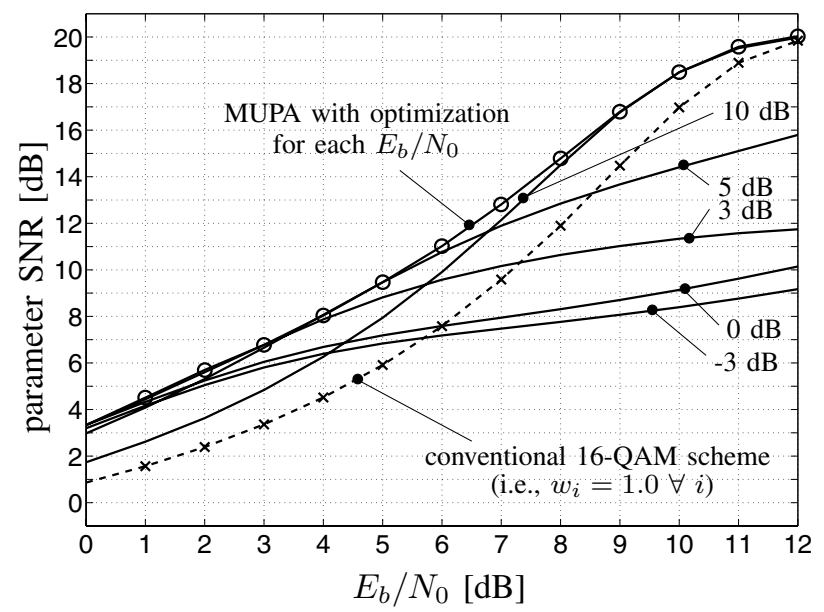

Fig. 9. Comparison of 16-QAM ( $\Gamma: \mathrm{NB}, \mu$ : Gray) with and without MUPA (analog to Fig. 8)

ing to the different significance of the transmitted bits. The analysis shows that the weights approach towards a fixed value at high $E_{b} / N_{0}$ values, while the weights diverge at low channel qualities. Parameter SNR gains of up to $2.61 \mathrm{~dB}$ for BPSK-MUPA and up to $3.55 \mathrm{~dB}$ for 16-QAM-MUPA are demonstrated in comparison to systems with constant symbol energy. Even with mismatched weights MUPA improves the system performance.

\section{REFERENCES}

[1] J. Hagenauer, "Rate-Compatible Punctured Convolutional Code (RCPC Codes) and their Applications," IEEE Transactions on Communications, pp. 389-400, Apr. 1988.

[2] T. Brüggen, C. Schulte-Hillen, and P. Vary, "Soft Demodulation and Unequal Error Protection for Digital Modulation Schemes," in Proc. of IEEE ICASSP, Philadelphia, PA, USA, Mar. 2005.

[3] T. Brüggen and P. Vary, "Unequal Error Protection by Modulation with Unequal Power Allocation," accepted for publication in IEEE Commun. Lett., 2005.

[4] E. Bedrosian, "Weighted PCM," IRE Transactions on Information Theory, vol. IT-4, pp. 45-49, Mar. 1958.

[5] N. Görtz and E. Bresch, "Source-Adaptive Power Allocation for Digital Modulation,” IEEE Commun. Lett., vol. 7, pp. 569-571, Dec. 2003.

[6] J. G. Proakis, Digital Communications. McGraw-Hill Inc., 1995.

[7] A. Papoulis and S. U. Pillai, Probability, Random Variables, and Stochastic Processes. McGraw-Hill Inc., 2002.

[8] W. T. Webb, L. Hanzo, Modern Quadrature Amplitude Modulation. Pentech Press, 1994. 